\title{
Review Article \\ Enkephalins: Endogenous Analgesics with an Emerging Role in Stress Resilience
}

\author{
Mathilde S. Henry, ${ }^{1}$ Louis Gendron, ${ }^{2,3}$ Marie-Eve Tremblay, ${ }^{1}$ and Guy Drolet ${ }^{1}$ \\ ${ }^{1}$ Axe Neurosciences, Centre de Recherche du CHU de Québec - Université Laval, Québec, QC, Canada \\ ${ }^{2}$ Département de pharmacologie-physiologie, Centre de Recherche du CHU de Sherbrooke, Institut de Pharmacologie de Sherbrooke, \\ Université de Sherbrooke, Sherbrooke, QC, Canada \\ ${ }^{3}$ Quebec Pain Research Network, Sherbrooke, QC, Canada
}

Correspondence should be addressed to Marie-Eve Tremblay; tremblay.marie-eve@crchudequebec.ulaval.ca

Received 14 March 2017; Revised 15 May 2017; Accepted 24 May 2017; Published 11 July 2017

Academic Editor: Azucena B. Losa

Copyright ( 2017 Mathilde S. Henry et al. This is an open access article distributed under the Creative Commons Attribution License, which permits unrestricted use, distribution, and reproduction in any medium, provided the original work is properly cited.

\begin{abstract}
Psychological stress is a state of mental or emotional strain or tension that results from adverse or demanding circumstances. Chronic stress is well known to induce anxiety disorders and major depression; it is also considered a risk factor for Alzheimer's disease. Stress resilience is a positive outcome that is associated with preserved cognition and healthy aging. Resilience presents psychological and biological characteristics intrinsic to an individual conferring protection against the development of psychopathologies in the face of adversity. How can we promote or improve resilience to chronic stress? Numerous studies have proposed mechanisms that could trigger this desirable process. The roles of enkephalin transmission in the control of pain, physiological functions, like respiration, and affective disorders have been studied for more than 30 years. However, their role in the resilience to chronic stress has received much less attention. This review presents the evidence for an emerging involvement of enkephalin signaling through its two associated opioid receptors, $\mu$ opioid peptide receptor and $\delta$ opioid peptide receptor, in the natural adaptation to stressful lifestyles.
\end{abstract}

\section{Introduction}

Psychological stress is a state of mental or emotional strain or tension that results from adverse or demanding circumstances. It has multifaceted causes and occurs frequently over a lifetime with varying dimensions and intensity, affecting all walks of life, irrespective of a person's occupation or position within a society [1]. While depression is often the devastating outcome of chronic stress [2] and also a risk factor and common comorbidity in Alzheimer's disease [3,4], stress resilience, on the other hand, is a positive outcome that is associated with preserved cognition, reduced oxidative damage, and healthy aging $[5,6]$. The American Psychological Association defines resilience as "the process of adapting well in the face of adversity, trauma, tragedy, threats or even significant sources of threat." Heterogeneity in the response to chronic stress suggests that resilience is a complex neurobiological process that emerges from a multitude of gene-environment interactions. Several mechanisms are proposed to underlie the interindividual differences in resilience or vulnerability to chronic stress.

Within the neuropeptidergic system, the endogenous opioids enkephalins (ENK) which signal through the opioid peptide receptors (OPr), $\mu$ opioid peptide receptor (MOPr) and $\delta$ opioid peptide receptor (DOPr), could be interesting candidates to naturally promote the adaptation to chronic stress. ENK are members of the endorphin family and the first ones to be isolated in the brain [7]. Considering the binding of morphine and ENK to the same receptors, their role as a natural analgesic was rapidly proposed. Pioneered studies have provided the first experimental evidence supporting a role of ENK in analgesia and stress-induced analgesia (i.e., pain suppression after an exposure to stressful stimuli). More specifically, it was shown in the rat that 1) 
the cerebroventricular injection of ENK produces analgesia $[8,9]$; 2) stress increases blood concentrations of ENK $[10]$; and 3) stress-induced analgesia, such as immobilization stress on a hot plate or cold water stress, could be reversed by an opioid antagonist $[11,12]$. Subsequently, it was hypothesized that ENK were playing a major role in stress processes independently of their analgesic functions. Madden et al. reported that inescapable stress induced by footshocks (mimicking a posttraumatic stress disorder; PTSD) increases brain levels of ENK [10]. Another study showed a decrease of ENK immunoreactivity in the rat hypothalamus (HPT) after stress induced by footshocks [13]. More recently, ENK in the rat amygdala (AMG) were implicated in Pavlovian conditioned fear $[14,15]$ as well as in various behavioral and neuroendocrine aspects of the stress response [16-18]. The ENK are known to be involved in a large set of physiological and emotional processes, but their role in the individual capacity for stress adaptation has received less interest. In this review, the biochemistry of ENK and their anatomical distribution within the central nervous system (CNS) will be described first, followed by coverage of the well-known functions of ENK in emotional behaviors, including their key involvement in Pavlovian conditioned fear, anxiety, and stress response. Subsequently, the emerging role of ENK in the development of stress resilience will be discussed, with an emphasis on the recruitment of ENK projections coming from the AMG. The AMG is considered a key brain structure mediating the regulation of emotions and affective behavior, and the role of ENK in the stress response is notably suggested by their extended distribution in the AMG.

\section{Enkephalins and Their Opioid Receptors}

2.1. Biochemistry and Anatomical Distribution of Enkephalins and Their Receptors, DOPr and MOPr, in the CNS. ENK are produced from a propeptide precursor, proenkephalin (proENK), which is translated from preproenkephalin mRNA that is encoded by a gene distinct from the other endogenous opioid peptides [19, 20]. The maturation of propeptides into functional peptides is performed during the vesicular transport within large dense-core vesicles (LDCVs) and requires the joint action of several endopeptidases (cathepsin L, aminopeptidase $\mathrm{B}$ and $\mathrm{E}$, and prohormone convertase 2) [21-23]. In the rat, the proENK is cleaved proteolitycally to produce four copies of methionine-ENK (Met-ENK), one leucine-ENK (Leu-ENK), and two Cterminal extended Met-ENK. Subsequently, LDCVs are stored near release sites (i.e., presynaptic, extrasynaptic, and dendritic) and released following an increase in intracellular calcium [24]. Once released by neurons, ENK are degraded in order to control the diffusion and synchrony of the signal. Some studies demonstrated that radioactively labeled ENK are completely degraded in less than a minute upon injection (intracerebroventricular) in the rat brain [25]. ENK degradation is performed by two neuropeptidases called metallopeptidases: aminopeptidase $\mathrm{N}$ and neutral endopeptidase (or neprilysin) [26, 27]. In vitro, ENK have a slightly higher affinity for DOPr, even though they can also bind and activate MOPr and $\kappa$ opioid peptide receptor
(KOPr) in transfected cells transiently expressing MOPr, DOPr, or KOPr [28]. Studies describing the distribution of ENK in the rat brain have demonstrated their preferential binding to DOPr and MOPr by autoradiographic labelling [29].

Given the vast extent of biological processes and physiological systems in which ENK are involved (cardiovascular system, thirst and feeding, pain and analgesia, gastrointestinal functions, respiration, etc. [30]), the expression of ENK, $\mathrm{DOPr}$, and MOPr is ubiquitous. Indeed, ENK are distributed among the central, peripheral, and autonomous nervous systems, as well as in endocrine tissues (adrenal medulla, endocrine pancreas) and their target organs (liver, skin, bones, and lungs) [31, 32]. For the purpose of this review, we will focus mainly on the neuroanatomical distribution of ENK and their receptors within the "emotional brain" known as the limbic system that includes the cingulate and entorhinal cortex, hippocampus (HPC), septum, HPT, and the extended AMG [33]. Most of neuroanatomic studies have been conducted in rats, although several studies have also been conducted in humans, showing a similar distribution across species, especially in the limbic system [34]. Fallon and Leslie extensively reported in 1986 the distribution of ENK neurons as well as ENK fibers in the rat brain using an indirect immunofluorescence technique [35]. ENK neurons are found among the entorhinal, piriform, and medial prefrontal cortex (mPFC, infralimbic and prelimbic). Most nuclei of the HPT were shown to contain ENK neurons (paraventricular, posterior, ventromedial, dorsal, dorsomedial, and lateral nuclei). They are widely distributed in the central (CEA), medial (MEA), and basolateral (BLA) AMG and its intercalated (IC) nuclei. ENK neurons are also located in the lateral septum, preoptic area, bed nuclei of the stria terminalis (BST), nucleus accumbens (NAc), and ventral tegmental area (VTA). In the HPC, ENK are present in mossy fibers and granular cells. ENK fibers mainly project from the dentate gyrus to the CA3 region of Ammon's horn, but also target some neurons of the CA1 and CA2, and dentate gyrus. Additionally, ENK fibers are found in the dorsal and ventral pallidum $[35,36]$.

Similar to ENK, OPr are extensively expressed throughout the CNS [37]. The anatomical distribution of MOPr and DOPr is relatively similar to that of ENK projections [38]. To study the relative distributions of MOPr and DOPr throughout the CNS, Scherrer and colleagues have generated a very useful mouse model. They first developed DOPr-eGFP knock in (KI) mice, presenting a complete functional receptor fused to an enhanced green fluorescent protein (eGFP) [39]. These mutant mice were subsequently crossed to another model containing a similar construct, MOPr-mcherry KI mice [40]. This breeding generated a double KI mouse useful for in situ visualization of DOPr and MOPr simultaneously $[40,41]$. The study of DOPr and MOPr distribution in the CNS showed that coexpression of DOPr and MOPr is observed in HPT, HPC, the lateral parabrachial nucleus and vestibular nuclei, circuitries which are involved in survival including water and food consumption, sexual behavior, and response to aversive stimuli [40]. The large distribution of ENK and their associated receptors in the limbic system 
of rodents and humans further suggests that ENK transmission plays a major role in emotional behaviors.

2.2. Roles in Emotional Behaviors. ENK are indeed involved in several emotional behaviors, including fear conditioning $[14,15,42-45]$, anxiety, and stress response [46-65]. This section will describe the experimental evidence for such a role, mainly derived from studies conducted in rodents, using different approaches, neuroanatomical, silencing, pharmacological, and genetic, as well as stress paradigms varying in chronicity and intensity.

2.2.1. Fear Conditioning. The fear conditioning paradigm allows assessment of learning and memory in association with fear (see Table 1). The first evidence that ENK participate in fear conditioning comes from an in situ hybridization study showing an increase in ENK mRNA levels in the CEA neurons of rats undergoing this paradigm [14]. Thereafter, it was shown that ENK knockout (KO) mice exhibit an exaggerated immobility compared to wild-type controls during the auditory-conditioned fear acquisition [42]. A population of GABAergic neurons expressing protein kinase C- $\delta(\mathrm{PKC}-\delta)$ was identified in the lateral part of CEA (CEAl), using a molecular genetic approach in mice. Interestingly, this population appears to overlap with ENK neurons [45]. In another study, it was shown that this neuronal population expressing PKC- $\delta$ in the CEAl is implicated in the inhibition of fear acquisition [66]. However, the exact role of ENK expressed by these PKC- $\delta$ GABAergic neurons is still undetermined.

Asok et al. also showed that exposure to a component of fox odor, 2,5-dihydro-2,4,5-trimethylthiazoline (TMT), which triggers innate fear in rats, increases ENK mRNA levels in the paraventricular nucleus (PVN) of the HPT [43]. An increased expression of ENK mRNA levels is similarly observed after repeated footshocks, in the AMG of SWR/J mice, an inbred strain showing a reduced fear response, while this expression was unchanged in C57Bl/6J mice, an inbred strain showing a high fear response [44]. In the same study, administration of MOPr antagonist (naltrexone) or DOPr antagonist (naltrindole) increased fear response in SWR/J mice, which could be restored with a DOPr agonist. These results suggest that resistance in the face of traumatic experiences inducing fear involves ENK from the AMG and that vulnerability can be modulated by administration of OPr agonists [44]. Finally, it has been shown by Poulin et al. that the downregulation of ENK in the rat CEA decreases unconditioned fear [15]. In this study, rats were submitted to a contextual conditioning paradigm consisting of footshocks administered in a novel environment. ENK knockdown (KD) rats showed a reduced fear response during conditioning, while the context alone, presented $48 \mathrm{~h}$ later, did not produce change in freezing behavior. These results indicate that ENK release from CEA neurons is involved in the freezing behavior to an unconditioned stimulus, but not in the formation of an associative memory [15]. Results of ENK distribution studies-in addition to pharmacological, silencing, and genetic studies-demonstrate the prominent role of ENK, especially amygdalar ENK, in mediating fear behavior. This connection may further suggest a role for ENK in anxiety and stress responses, which are closely related to fear behavior.

2.2.2. Stress and Anxiety. Several studies performed in humans showed the importance of ENK in anxiety, depression, and PTSD, a mental illness that appears after experiencing a traumatic event. Indeed, a polymorphism in the gene encoding neutral endopeptidase, involved in ENK metabolism, was identified in patients with anxiety disorder, tested with the SCL-90-R inventory of psychological symptoms [48]. Positron emission tomography (PET) studies have shown that MOPr expression is decreased in the anterior cingulate cortex of patients with PTSD [47]. In patients with depression, PET further revealed that the expression of MOPr is decreased in the HPT and AMG [49]. These studies suggest that a reduced tone of ENK neurotransmission is a key component in the expression of anxiety.

In rodents, several behavioral paradigms are commonly used to assess the level of anxiety, including the elevated plus maze (EPM), open field (OF), and light-dark box (LDB) tests. These tests are based on the natural aversion of rodents for open, elevated, or illuminated areas and their natural exploratory behavior in novel environments. In addition, the social interaction test (SI) allows evaluating the propensity to socialize. The startle response (SR) corresponds to an unconscious defensive response to unexpected or threatening stimuli. All behavioral tests discussed in our review are detailed in Table 1.

The ENK KO mice show an increased anxiety with the EPM, OF, and LDB tests, have an exaggerated SR, and a reduced duration of SI $[42,50,51]$. ENK KO mice exposed to a stress induced by footshocks, mimicking PTSD, similarly present anxiety- and depressive-like behaviors, contrary to wild-type controls, using the OF, EPM, and LDB tests (see Table 1) [52]. However, the downregulation of ENK in CEA was shown to reduce anxiety as characterized by an increase of exploratory behavior [15]. ENK KO mice are resistant to anxiety- and depression-like behaviors after a chronic mild unpredictable stress-consisting of daily exposure to different stressors, such as food deprivation and restraint stress for five weeks-suggesting that ENK enhance the reactivity to chronic stress [67]. ENK appear to have varying and even opposing effects on anxiety, depending on the considered CNS region and the type and intensity of stress.

The high levels of anxiety generally observed in ENK KO mice are also seen upon gene inactivation of DOPr [53]. Pharmacological studies conducted in rodents support these results obtained through gene inactivation of DOPr, since subcutaneous administration of naltrindole, a DOPr antagonist, induces anxiety [54]. Conversely, intraperitoneal injection of DOPr agonists (SNC80, UFP-512, (+)BW373U86) was shown to be anxiolytic [55-57]. Moreover, infusion of [D-Pen 2,5]-ENK (DPDPE), a DOPr agonist, in CEA exerted similar effects, which could be reversed by the administration of naltrindole, a DOPr antagonist. Recently, a new DOPr agonist, KNT-127, has received an increasing interest as a potential therapeutic treatment for anxiety and depression, although the efficacy of this molecule has not yet been 
TABLE 1: Evidence for ENK signaling involvement using different behavioral tests.

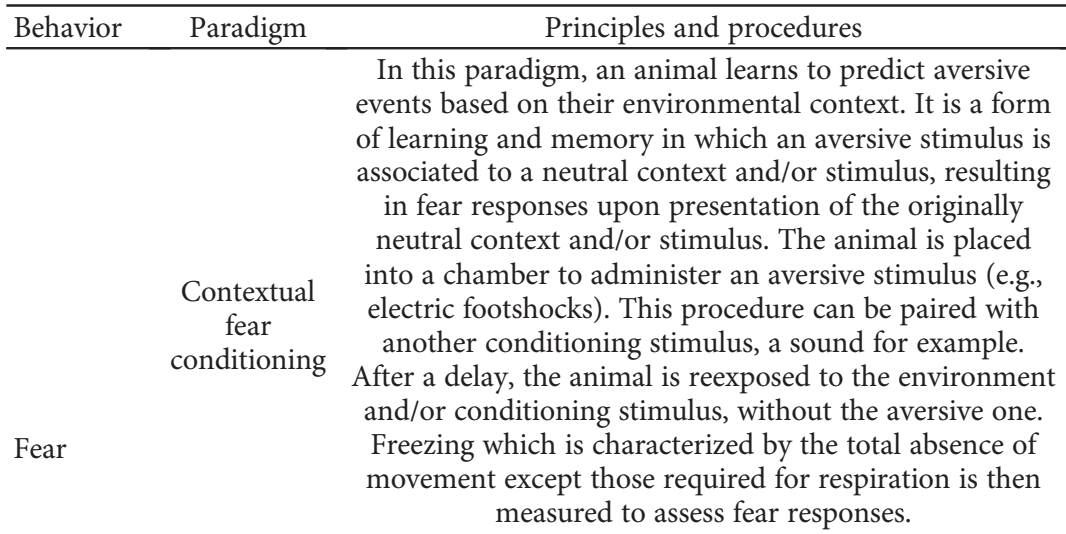

The startle reflex is considered as an innate and involuntary reaction that appears upon exposure to an unexpected or threatening stimuli. The response Startle corresponds to a quick involuntary contraction of the response animal's skeletal muscles. The test is conducted in an automated startle chamber that allows measurement of the reflex.

This task is based on a rodent's preference for dark areas. The animal is placed in an open-field chamber, an arena with surrounding walls to prevent escape, and the exploratory behavior of the center (lit) versus periphery (dark) is assessed over time with a video-recording.

Elevated plus maze

Anxiety

Open-field

plus maze

This task is based on a rodent's natural preference for dark and enclosed areas, compared to lit and uncovered areas, as well as on their natural exploratory behavior of a novel environment. The animal is placed in the maze, and its exploratory behavior is assessed over time with a video-recording. The maze has a cross shape with two opposite arms surrounded by walls (dark and enclosed area) whereas the two other arms do not present walls (lit and uncovered).

This task is based on a rodent's natural preference for dark areas, compared to lit ones. The box contains two

Light-dark box

Social interaction test chambers, one light and one dark. The animal is placed into the box and its exploratory behavior is assessed over time with a video-recording.

This test allows evaluating the propensity of an individual to socialize. The rodent is placed in an open-field arena alone in the first place and then with another individual. The time spent interacting with the intruder is measured.
Evidence for involvement of ENK signaling

(i) In rats, ENK mRNA levels are increased in CEA upon contextual fear conditioning [14]

(ii) ENK knockout $(\mathrm{KO})$ mice show an exaggerated immobility during auditory fear conditioning [42]

(iii) ENK neurons in CEAl overlap with $\mathrm{PKC}-\delta$ GABAergic neurons, which are involved in fear behavior $[45,66]$

(iv) In SWR/J mice (showing a reduced fear response induced by footshocks), ENK mRNA levels are increased in AMG [44]

(v) In SWR/J mice (showing a reduced fear response induced by footshocks), administration of MOPr and DOPr antagonists increase fear response [44]

(vi) In rats, ENK knockdown (KD) of CEA decreased unconditioned fear [15].

(i) ENK KO mice show an exaggerated startle response [50].

(i) ENK KO mice show a decreased exploratory behavior and avoid the central part of the open-field (OF) arena $[42,50,51]$

(ii) ENK KO mice, exposed to stress induced by footshocks, present an anxiety-like behavior [52].

(i) ENK KO mice present anxiety-like behavior in the elevated plus maze (EPM) [50]

(ii) ENK KO mice, exposed to stress induced by footshocks, present anxiety-like behavior in EPM [52]

(iii) In rats, ENK KD in CEA increases the exploratory behavior in EPM [15]

(iv) Infusion of a DOPr agonist in CEA increases the number of entries and the time spent in open arms of the EPM [82]

(v) Administration of a DOPr antagonist diminishes the exploratory behavior in EPM [54]

(vi) Administration of a DOPr agonist increases this behavior $[55-57,59,60]$

(vii) DOPr KO mice spent less time in the open arms of EPM [53]

(viii) MOPr KO mice increase the exploratory behavior in EPM [53]

(ix) Administration of MOPr agonist increases the exploratory behavior in EPM [58].

(i) ENK KO mice show a decreased exploratory behavior in the light-dark box (LDB) $[42,50]$

(ii) ENK KO mice, exposed to stress induced by footshocks, present an anxiety-like behavior in LDB [52]

(iii) $\mathrm{DOPr} \mathrm{KO}$ mice spent less time in the illuminated portions of the LDB [53].

(i) ENK KO mice present a reduced duration of social interaction [50]. 
TABle 1: Continued.

\begin{tabular}{lccc}
\hline Behavior & Paradigm & Principles and procedures & Evidence for involvement of ENK signaling \\
\hline $\begin{array}{c}\text { Forced swim } \\
\text { test }\end{array}$ & $\begin{array}{c}\text { This test is used to evaluate the antidepressant } \\
\text { efficacy of new compounds. A rodent is placed in } \\
\text { a pool containing approximately 15 } \mathrm{cm}^{3} \text { of water, } \\
\text { and its mobility is measured on a video-recording. }\end{array}$ & $\begin{array}{c}\text { (i) Administration of a DOPr agonist increases } \\
\text { mobility in the forced swim test [59]. }\end{array}$ \\
Anhedonia & $\begin{array}{c}\text { Sucrose } \\
\text { preference } \\
\text { test }\end{array}$ & $\begin{array}{c}\text { This task is used as an indicator of anhedonia, } \\
\text { characterized by a lack of interest for a reward. Two } \\
\text { bottles, one containing a sucrose solution (between 1\% } \\
\text { and 5\%) and another plain water, are presented to the } \\
\text { animal. Its preference for the sweetened versus plain } \\
\text { water reveals anhedonia state }\end{array}$ & $\begin{array}{c}\text { (i) After restraint stress, rats showing increased } \\
\text { anhedonia (assessed with the sucrose preference } \\
\text { test) present a reduced expression of ENK } \\
\text { mRNA in the NAc [70]. }\end{array}$ \\
\hline
\end{tabular}

investigated in clinical trials. In rodents, KNT-127 produces anxiolytic and antidepressant-like effects in a dosedependent manner (see Table 1) $[59,60]$. These results are consistent between models and suggest that signaling onto DOPr mainly exerts anxiolytic effects.

In contrast to these findings, a conditional $\mathrm{KO}$ mouse for DOPr (Dlx-DOR) in forebrain GABAergic neurons showed a reduced level of anxiety compared to wild-type littermates, demonstrating that stimulation of DOPr in GABAergic neurons of the forebrain is anxiogenic (see Table 1) [62]. In the same way, the gene inactivation of MOPr has anxiolytic effects, with MOPr KO mice presenting an increased time spent in the open arms of an EPM [53]. Nevertheless, several pharmacological studies instead demonstrated that MOPr activation is anxiolytic. For example, intraperitoneal administration of morphine, a MOPr agonist, decreases vocalizations in rats exposed to a predator and anxiety assessed with the EPM test [58]. Overall, MOPr appear to have varying effects on anxiety, depending on the methodological approaches used.

A few recent studies explored the neuroanatomical specificity of ENK projections that are recruited in steady-state conditions or upon stress in rats. Single housing (see Table 2) in early life was shown to decrease immunoreactivity of Met-ENK-Arg ${ }^{6} \mathrm{Phe}^{7}$ (MEAP) in the brain areas that include the AMG, substantia nigra (SN), HPT, and periaqueductal grey (PAG) [63]. Hernández et al. also measured ENK neuropeptidase activities in the three main regions of the stress response circuitry (AMG, HPC, and mPFC) after acute restraint in rats (see Table 2; [46]). Neuropeptidases regulate the expression of neuropeptides at the release sites. Peptidase activity can thus be used to indicate the functional status of neuropeptides. This neuropeptidase activity was found to be more intense in AMG than in HPC or mPFC both in control and stressful conditions, suggesting that ENK metabolism is preponderant in the AMG. After acute restraint stress, ENK-degrading activity was reduced in AMG and increased in HPC, while it remained unchanged in the mPFC. In stressed rats, a positive correlation was described between the AMG and HPC, while in control rats, a negative correlation was observed between the MPFC and HPC. These results suggest a neuropeptidergic functional connection between the mPFC, HPC, and AMG, which could be triggered by stress and involved in some of the adaptive functions performed by this circuit.
Overall, these contradictory results found in the literature regarding the influence of ENK signaling on anxiety could be attributed first, to the technical approaches (pharmacological, genetic), then to the considered nucleus (CEA for example) or associated neurotransmitters (GABA), and finally to the type (acute, chronic stress) and intensity of stress. It still remains unknown whether the many effects of ENK circuitry acting in such a diverse array of brain circuits might all be recruited together in response to a variety of different stressors and different modalities. Different brain circuits could synergistically contribute to the stress response, highlighting the huge challenge we face in understanding the functions of ENK signaling. Taken together, the combined findings from these silencing, pharmacological, genetic, and neuroanatomical studies suggest that the stimulation of ENK transmission onto DOPr and/or MOPr might enhance the natural strategies to cope with stress.

\section{Enkephalin Signaling through DOPr and MOPr, a Major Component of the Stress Resilience Circuitry}

An extreme amount of stress can lead to maladaptive behavioral changes such as anhedonia and social avoidance, in rodents and humans, as well as serious health consequences by impacting on the nervous, endocrine, and immune systems. However, chronic exposure to stress can also engender compensatory physiological responses in order to reduce these deleterious effects of stress. This mechanism of defense allows maintaining homeostasis in the face of adversity. This phenomenon of "resilience" corresponds to the ability of an individual to maintain normal psychological and physical functioning in the front of stress or trauma, in order to avoid mental and physical illnesses [68].

Recent findings regarding the functions of ENK transmission in stress resilience revealed the involvement of different brain areas such as the NAc $[69,70]$ or septum, PVN and PAG $[17,18,71]$, or locus coeruleus (LC) and paragigantocellularis nucleus (PGi) [72], in addition to the BLA as we will discuss below, thus suggesting a high level complexity of ENK circuitry in stress resilience.

Sweis et al. associated the resilience to chronic stress-measured by a lack of memory impairment poststress-to an increased expression of ENK mRNA in the 
TABLE 2: Evidence for ENK signaling involvement under different stress paradigms.

\begin{tabular}{lcc}
\hline Paradigm & Principles and procedures & Evidence for involvement of ENK signaling \\
\hline Single housing & $\begin{array}{c}\text { Given the social behavior of rodents, chronic or acute single } \\
\text { housing is used to mimic the stress due to social isolation. } \\
\text { The animal is placed alone in its home cage. }\end{array}$ & $\begin{array}{c}\text { (i) Prolonged single housing in early life decreases ENK } \\
\text { immunoreactivity in AMG, SN, HPT, and PAG [63]. }\end{array}$ \\
\hline
\end{tabular}

(i) After acute restraint stress, ENK-degrading activity is reduced in AMG and increased in HPC [46]

The animal is placed in a tube in such a way that all movements are prevented. The psychological and physiological effects due to restraint stress result from the distress and aversive nature of the forced immobility. (ii) After chronic restraint stress, ENK knockout (KO) mice do not exhibit anxiety nor depression-like behavior [67]

(iii) After chronic restraint stress, rats showing increased anhedonia present a reduced expression of ENK mRNA in the NAc [70].

(i) After a chronic social defeat, Oprm1 A112G mice show a strong resilience [71]

(ii) After a chronic social defeat, resilient rats demonstrate a high recruitment of ENK afferents from PGi to LC [72]

(iii) After a chronic social defeat in rats and mice, ENK mRNA levels decrease in BLA of vulnerable individuals $[17,81]$.

(i) ENK knockdown (KD) in BLA increases anxiety reproducing behavioral responses encountered in individuals vulnerable to chronic unpredictable stress [18].
Chronic unpredictable stress
This test allows to mimic the unpredictable disruptions of daily life. An animal is subjected to different stressors each day for a given period of time. Stressors can include restraint stress, electric footschocks, wet bedding, group housing, mild

shaking of the home cage, cold water swim, etc. victimization in humans. An intruder is placed in the home cage of a resident each day for a given period of time.

This task exploits the social conflict between two individuals to initiate psychological stress. This Social defeat stress (or resident-intruder paradigm) over a given period of time, induces stress resilience by mimicking the unpredictable social disruptions of daily life. This paradigm has been shown to present excellent etiological, predictive, discriminative, and face validity [75]. Moreover, unlike other stress paradigm, social defeat stress leads to long-lasting changes in hypothalamic-pituitary-adrenal axis function, making it a stress paradigm of choice [76]. The majority of rodents exposed to this paradigm exhibits reduced motivation, anhedonia, and avoids social interactions [77]. Conversely, despite the deleterious effects of social stress, around $30 \%$ of the population presents a phenotype of stress resilience, being resistant to the emergence of depressive-like behavior. In rats, the daily interaction between individuals results in subordination of the intruder, indicated by adoption of a supine position. The latency to assume a defeated posture is recorded, and the averaged latency over stress exposition is used as a predictive value to define resilience or vulnerability to stress. In mice, the resilience or vulnerability to stress is instead assessed at the end of this experiment by using a SI test (see Table 1). The second chronic stress paradigm that is commonly used to study stress resilience is chronic unpredictable stress. In this experiment, individuals are daily submitted to different stressors that include restraint stress, wet bedding, food deprivation, and footshocks. The phenotype of resilience or vulnerability to stress is assessed at the end of the experiment in mice and rats using behavioral tests previously described such as SI, EPM, and OF (also see Table 1).

For example, Briand et al. used the repeated social defeat paradigm in a mouse model of OPRM1 A118G polymorphism (single nucleotide polymorphism, SNP) correspond- ing to a genetic mutation of MOPr observed in humans paradigm, in which intruder animals are repeatedly submitted to daily interactions with a home-cage unfamiliar resident rat NAc, proposing that an ENK-mediated increase of dopaminergic tone could improve motivation-based cognitive performance [69]. This predominant role of ENK proanother study. Indeed, it was shown that after 14 days of restraint stress, rats showing increased anhedonia (as meapresented in the NAc a reduced expression of ENK mRNA and $\triangle$ FosB, a transcription factor that is expressed by ENK neurons. These results suggest that the individual vulnerabilnia, is associated with a $\Delta$ FosB-mediated downregulation of ENK [70]. The relationship between $\Delta$ FosB and the resilience chronic stress was already known [73]. effects of dominance status and housing conditions on the that activation in the mPFC, CEA, and NAc. Triad housing for the same period of time also increases DOPr activation in resilient ind and frequent aggressive interactions with $\alpha$ dominant rats, which instead display an offensive behavior [74]. This mechanism could be involved in the regulation of ENK transmission upon stress.

Two types of behavioral paradigms are commonly conducted in rodents for studying stress resilience (see Table 2). The social defeat paradigm, also named resident-intruder 


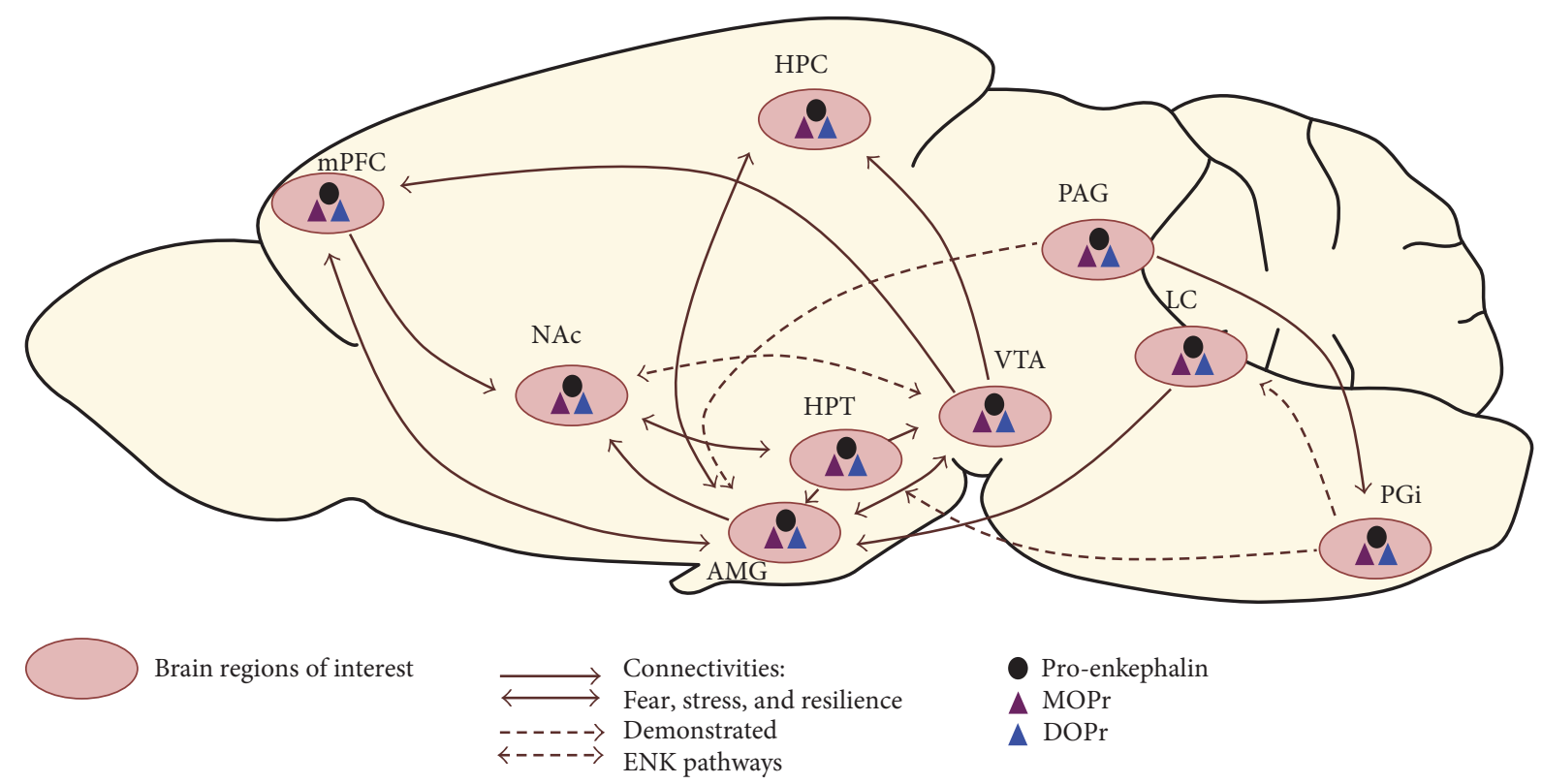

FIGURE 1: Cartography of main connectivities involved in fear, stress, and resilience, as well as demonstrated ENK pathways between areas and expression of ENK, MOPr, and DOPr. Pink circles represent brain regions of interest. Full arrows correspond to circuitries of stress, fear, and resilience. Dotted arrows represent demonstrated ENK circuitries. The black dot corresponds to expression of pro-enkephalin, and purple and blue triangles correspond to MOPr and DOPr expression, respectively. AMG: amygdala; HPC: hippocampus; HPT: hypothalamus; LC: locus ceruleus; mPFC: medial prefrontal cortex; NAc: nucleus accumbens; PAG: periaqueducal grey; PGi: paragigantocellularis nucleus; VTA: ventral tegmental area.

that is associated with an overall reduction of baseline MOPr availability in regions implicated in pain and affective regulation [78], thus allowing to unravel a potential role of MOPr in the resilience to chronic stress [71]. This model presents increased home-cage dominance and nonaggressive social interactions, similar to the human carriers of this mutation. In the presence of an aggressor during social defeat stress, it also showed a strong resilience to chronic stress, determined by a blunted anhedonia and social avoidance following the social defeat. Neuronal activation measured by c-fos staining was additionally increased in the NAc, septum, BLA, PVN, and PAG, thus suggesting an increased release of endogenous opioids upon stress [71]. In humans, Troisi et al. demonstrated that the carriers of this mutation have a greater capacity to experience social reward and are more prone to fearful attachment, a personality trait that is related to rejection sensitivity, regardless of the quality of maternal care [79, 80].

Reyes et al. also revealed involvement of the ENK circuitry between the LC and PGi in stress resilience in rats. In this study, fluorogold, a retrograde tracer, was injected into the LC to determine involvement of different afferents (corticotropin-releasing factor, CRF neurons from CEA and ENK neurons from PGi) in resilience under the residentintruder paradigm [72]. Individuals presenting a reduced latency to present a defeated posture (defined as vulnerable rats) showed an increased activation of LC neurons and afferents of CRF neurons from CEA. Conversely, resilient rats (longer latency to present a defeated posture) demonstrated a higher recruitment of ENK afferents from PGi. Thus, two different afferent pathways to the LC, from CRF neurons in the CEA and ENK neurons from the PGi, would partly define the interindividual variation with regard to the capacity to resist chronic stress.

Two studies conducted in our laboratory demonstrated that resilience to social defeat and chronic unpredictable stress share common variations of expression among the ENK systems within specific brain regions in rats $[17,18]$. ENK mRNA (transcripts) were quantified in 23 nuclei of the mPFC, NAc, dorsal striatum, and AMG. Only one significant difference between control, resilient, and vulnerable individuals was found in the BLA of vulnerable individuals; ENK mRNA levels were decreased in vulnerable rats compared to control and resilient rats. In contrast, no difference was found in ENK expression in the BLA between controls and resilient animals [17]. In addition to revealing these associations, the functional role of ENK in the AMG was evaluated. The downregulation of ENK in the BLA was shown to increase anxiety both in the SI test and EPM thus reproducing certain behavioral responses encountered in individuals that are vulnerable to chronic stress [18]. Finally, the chronic social defeat stress was conducted in mice in order to assess ENK signature in the BLA. The expression of ENK mRNA was found to be decreased by $33 \%$ in vulnerable mice, only in the BLA. No difference was found between the control and resilient individuals [81]. These combined results suggest that specific neuroadaptations mediated by ENK neurotransmission in the BLA could represent a key mediator of stress resilience. Based on these results, we can hypothesize that 
the decrease in ENK transmission from the BLA is a maladaptive mechanism, which mediates the behavioral dichotomy observed between vulnerable and resilient animals experiencing chronic stress.

\section{Conclusion}

Overall, most of animal studies covered in this review suggest that ENK signaling could be targeted for promoting resilience to chronic stress. Resilience to chronic stress is a very complex process involving several brain structures and neurotransmitters. When considering only one neuropeptidergic system, the ENK acting through DOPr and MOPr, numerous implicated brain structures and circuits emerge (see Figure 1 for a schematic representation that we overlapped with the cartography of main connectivities known to be involved in stress response, fear, and resilience). While the roles of ENK signaling within certain brain structures such as AMG, HPT, and NAc were largely described, its involvement in other brain regions remains unknown with regard to stress resilience. For example, the preoptic area, BST, and piriform cortex express ENK without evidence for a potential role in resilience to chronic stress, to our knowledge. All of these circuits must be individually dissected. Complete ENK KO models may thus be inadequate for characterizing the involvement of ENK signaling in stress resilience. Hence, modulating ENK or DOPr/MOPr expression within circumscribed regions or modulating selected neuronal circuits appear to be more appropriate. In this regard, optogenetic tools could provide a unique opportunity to modulate ENK transmission among selected neuronal circuits, over the course of chronic stress and associated pathologies, as required to unravel the mechanisms through which distinct ENK pathways exert their functional role in stress resilience. Understanding the synergistic involvement of different circuits in stress resilience could additionally provide accurate, powerful, and effective therapeutic strategies to prevent or treat long-term anxiety and depression, in addition to a variety of stress- and anxiety-related disorders.

\section{Abbreviations}

DPDPE: [D-Pen 2,5]-enkephalin

TMT: 2,5-Dihydro-2,4,5-trimethylthiazoline

AMG: Amygdala

BLA: Basolateral nucleus of amygdala

BST: $\quad$ Bed nuclei of the stria terminalis

CNS: Central nervous system

CEA: Central part of amygdala

CRF: Corticotropin-releasing factor

EPM: $\quad$ Elevated plus maze

ENK: Enkephalin

FST: $\quad$ Forced swim test

HPC: Hippocampus

HPT: Hypothalamus

IC: $\quad$ Intercalated nuclei of amygdala

KD: $\quad$ Knock down

KI: $\quad$ Knock in
KO: $\quad$ Knockout

LDCVs: Large dense-core vesicles

CEAl: $\quad$ Lateral part of central amygdala

Leu-ENK: Leucine-enkephalin

LC: $\quad$ Locus ceruleus

MEA: $\quad$ Medial part of amygdala

mPFC: $\quad$ Medial prefrontal cortex

MEAP: $\quad$ Met-ENK-Arg ${ }^{6} \mathrm{Phe}^{7}$

Met-ENK: Methionine-enkephalin

NAc: $\quad$ Nucleus accumbens

OF: Open-field

LDB: Light-dark box

OPr: $\quad$ Opioid peptide receptor

PGi: Paragigantocellularis nucleus

PVN: $\quad$ Paraventricular nucleus of HPT

PAG: $\quad$ Periaqueducal grey

PET: $\quad$ Positron emission tomography

PTSD: $\quad$ Posttraumatic stress disorder

Pro-ENK: Proenkephalin

PKC- $\delta$ : Protein kinase C- $\delta$

SI: $\quad$ Social interaction

SN: $\quad$ Substantia nigra

SR: $\quad$ Startle response

VTA: Ventral tegmental area

DOPr: $\quad \delta$ opioid peptide receptor

KOPr: $\quad \kappa$ opioid peptide receptor

MOPr: $\quad \mu$ opioid peptide receptor.

\section{Conflicts of Interest}

The authors declare that they have no conflicts of interest.

\section{Authors' Contributions}

Marie-Eve Tremblay and Guy Drolet contributed equally to this work.

\section{Acknowledgments}

This study was supported by grants from the Canadian Institutes of Health Research (CIHR) attributed to Guy Drolet and Marie-Eve Tremblay. Louis Gendron holds a Chercheur boursier, senior scholar, and Marie-Eve Tremblay a Chercheur boursier, junior 1 scholar, from the FRQ-S, and a Canada Research Chair-Tier 2 in Neuroimmune Plasticity in Health and Therapy.

\section{References}

[1] A. Machado, A. J. Herrera, R. M. de Pablos et al., "Chronic stress as a risk factor for Alzheimer's disease," Reviews in the Neurosciences, vol. 25, no. 6, pp. 785-804, 2014.

[2] R. J. Davidson and B. S. McEwen, "Social influences on neuroplasticity: stress and interventions to promote well-being," Nature Neuroscience, vol. 15, no. 5, pp. 689-695, 2012.

[3] R. C. Green, L. A. Cupples, A. Kurz et al., "Depression as a risk factor for Alzheimer disease: the MIRAGE study," Archives of Neurology, vol. 60, no. 5, pp. 753-759, 2003. 
[4] P. J. Modrego, "Depression in Alzheimer's disease. Pathophysiology, diagnosis, and treatment," Journal of Alzheimer's Disease, vol. 21, no. 4, pp. 1077-1087, 2010.

[5] D. S. Charney, "Psychobiological mechanisms of resilience and vulnerability: implications for successful adaptation to extreme stress," The American Journal of Psychiatry, vol. 161, no. 2, pp. 195-216, 2004.

[6] M. L. Pfau and S. J. Russo, "Peripheral and central mechanisms of stress resilience," Neurobiology of stress, vol. 1, pp. 66-79, 2015.

[7] J. Hughes, T. W. Smith, H. W. Kosterlitz, L. A. Fothergill, B. A. Morgan, and H. R. Morris, "Identification of two related pentapeptides from the brain with potent opiate agonist activity," Nature, vol. 258, no. 5536, pp. 577-580, 1975.

[8] J. D. Belluzzi, N. Grant, V. Garsky, D. Sarantakis, C. D. Wise, and L. Stein, "Analgesia induced in vivo by central administration of enkephalin in rat," Nature, vol. 260, no. 5552, pp. 625-626, 1976.

[9] A. Pert, R. Simantov, and S. H. Snyder, "A morphine-like factor in mammalian brain: analgesic activity in rats," Brain Research, vol. 136, no. 3, pp. 523-533, 1977.

[10] J. Madden 4th, H. Akil, R. L. Patrick, and J. D. Barchas, "Stressinduced parallel changes in central opioid levels and pain responsiveness in the rat," Nature, vol. 265, no. 5592, pp. 358-360, 1977.

[11] S. Amir and Z. Amit, "Endogenous opioid ligands may mediate stress-induced changes in the affective properties of pain related behavior in rats," Life Sciences, vol. 23, no. 11, pp. 1143-1151, 1978.

[12] R. J. Bodnar, D. D. Kelly, A. Spiaggia, C. Ehrenberg, and M. Glusman, "Dose-dependent reductions by naloxone of analgesia induced by cold-water stress," Pharmacology, Biochemistry, and Behavior, vol. 8, no. 6, pp. 667-672, 1978.

[13] J. Rossier, R. Guillemin, and F. Bloom, "Foot shock induced stress decreases leu5-enkephalin immunoreactivity in rat hypothalamus," European Journal of Pharmacology, vol. 48, no. 4, pp. 465-466, 1978.

[14] G. D. Petrovich, A. P. Scicli, R. F. Thompson, and L. W. Swanson, "Associative fear conditioning of enkephalin mRNA levels in central amygdalar neurons," Behavioral Neuroscience, vol. 114, no. 4, pp. 681-686, 2000.

[15] J. F. Poulin, P. Bérubé, S. Laforest, and G. Drolet, “Enkephalin knockdown in the central amygdala nucleus reduces unconditioned fear and anxiety," The European Journal of Neuroscience, vol. 37, no. 8, pp. 1357-1367, 2013.

[16] A. L. Hebb, R. M. Zacharko, M. Gauthier, F. Trudel, S. Laforest, and G. Drolet, "Brief exposure to predator odor and resultant anxiety enhances mesocorticolimbic activity and enkephalin expression in CD-1 mice," The European Journal of Neuroscience, vol. 20, no. 9, pp. 2415-2429, 2004.

[17] P. Bérubé, S. Laforest, S. Bhatnagar, and G. Drolet, "Enkephalin and dynorphin mRNA expression are associated with resilience or vulnerability to chronic social defeat stress," Physiology \& Behavior, vol. 122, pp. 237-245, 2013.

[18] P. Bérubé, J. F. Poulin, S. Laforest, and G. Drolet, "Enkephalin knockdown in the basolateral amygdala reproduces vulnerable anxiety-like responses to chronic unpredictable stress," Neuropsychopharmacology, vol. 39, no. 5, pp. 11591168, 2014.
[19] H. Kakidani, Y. Furutani, H. Takahashi et al., "Cloning and sequence analysis of cDNA for porcine beta-neoendorphin/dynorphin precursor," Nature, vol. 298, no. 5871, pp. 245-249, 1982.

[20] M. Noda, Y. Furutani, H. Takahashi et al., "Cloning and sequence analysis of cDNA for bovine adrenal preproenkephalin," Nature, vol. 295, no. 5846, pp. 202-206, 1982.

[21] T. Hökfelt, C. Broberger, Z. Q. Xu, V. Sergeyev, R. Ubink, and M. Diez, "Neuropeptides-an overview," Neuropharmacology, vol. 39, no. 8, pp. 1337-1356, 2000.

[22] N. G. Seidah and M. Chretien, "Eukaryotic protein processing: endoproteolysis of precursor proteins," Current Opinion in Biotechnology, vol. 8, no. 5, pp. 602-607, 1997.

[23] D. F. Steiner, "The proprotein convertases," Current Opinion in Chemical Biology, vol. 2, no. 1, pp. 31-39, 1998.

[24] T. C. Suudhof, "Neurotransmitter release," Handbook of Experimental Pharmacology, vol. 184, pp. 1-21, 2008.

[25] F. Noble and B. P. Roques, "Protection of endogenous enkephalin catabolism as natural approach to novel analgesic and antidepressant drugs," Expert Opinion on Therapeutic Targets, vol. 11, no. 2, pp. 145-159, 2007.

[26] G. Waksman, E. Hamel, M. C. Fournié-Zaluski, and B. P. Roques, "Autoradiographic comparison of the distribution of the neutral endopeptidase "enkephalinase" and of mu and delta opioid receptors in rat brain," Proceedings of the National Academy of Sciences of the United States of America, vol. 83, no. 5, pp. 1523-1527, 1986.

[27] F. Noble, G. Banisadr, F. Jardinaud et al., "First discrete autoradiographic distribution of aminopeptidase $\mathrm{N}$ in various structures of rat brain and spinal cord using the selective iodinated inhibitor [ $\left.{ }^{125} \mathrm{I}\right] \mathrm{RB}$ 129," Neuroscience, vol. 105, no. 2, pp. 479-488, 2001.

[28] A. Mansour, M. T. Hoversten, L. P. Taylor, S. J. Watson, and H. Akil, "The cloned mu, delta and kappa receptors and their endogenous ligands: evidence for two opioid peptide recognition cores," Brain Research, vol. 700, no. 1-2, pp. 89-98, 1995.

[29] A. Mansour, M. E. Lewis, H. Khachaturian, H. Akil, and S. J. Watson, "Pharmacological and anatomical evidence of selective mu, delta, and kappa opioid receptor binding in rat brain," Brain Research, vol. 399, no. 1, pp. 69-79, 1986.

[30] R. J. Bodnar, "Endogenous opiates and behavior: 2015," Peptides, vol. 88, pp. 126-188, 2017.

[31] L. E. Eiden, "The enkephalin-containing cell: strategies for polypeptide synthesis and secretion throughout the neuroendocrine system," Cellular and Molecular Neurobiology, vol. 7, no. 4, pp. 339-352, 1987.

[32] G. M. Denning, L. W. Ackermann, T. J. Barna et al., "Proenkephalin expression and enkephalin release are widely observed in non-neuronal tissues," Peptides, vol. 29, no. 1, pp. 83-92, 2008.

[33] G. Drolet, E. C. Dumont, I. Gosselin, R. Kinkead, S. Laforest, and J. F. Trottier, "Role of endogenous opioid system in the regulation of the stress response," Progress in NeuroPsychopharmacology \& Biological Psychiatry, vol. 25, no. 4, pp. 729-741, 2001.

[34] Y. L. Hurd, "Differential messenger RNA expression of prodynorphin and proenkephalin in the human brain," Neuroscience, vol. 72, no. 3, pp. 767-783, 1996.

[35] J. H. Fallon and F. M. Leslie, "Distribution of dynorphin and enkephalin peptides in the rat brain," The Journal of Comparative Neurology, vol. 249, no. 3, pp. 293-336, 1986. 
[36] C. Gall, N. Brecha, H. J. Karten, and K. J. Chang, "Localization of enkephalin-like immunoreactivity to identified axonal and neuronal populations of the rat hippocampus," The Journal of Comparative Neurology, vol. 198, no. 2, pp. 335-350, 1981.

[37] J. Le Merrer, J. A. Becker, K. Befort, and B. L. Kieffer, "Reward processing by the opioid system in the brain," Physiological Reviews, vol. 89, no. 4, pp. 1379-1412, 2009.

[38] A. Mansour, H. Khachaturian, M. E. Lewis, H. Akil, and S. J. Watson, "Anatomy of CNS opioid receptors," Trends in Neurosciences, vol. 11, no. 7, pp. 308-314, 1988.

[39] G. Scherrer, P. Tryoen-Tóth, D. Filliol et al., "Knockin mice expressing fluorescent delta-opioid receptors uncover G protein-coupled receptor dynamics in vivo," Proceedings of the National Academy of Sciences of the United States of America, vol. 103, no. 25, pp. 9691-9696, 2006.

[40] E. Erbs, L. Faget, G. Scherrer et al., "A mu-delta opioid receptor brain atlas reveals neuronal co-occurrence in subcortical networks," Brain Structure \& Function, vol. 220, no. 2, pp. 677-702, 2015.

[41] E. Erbs, L. Faget, G. Scherrer et al., "Distribution of delta opioid receptor-expressing neurons in the mouse hippocampus," Neuroscience, vol. 221, pp. 203-213, 2012.

[42] A. Ragnauth, A. Schuller, M. Morgan et al., "Female preproenkephalin-knockout mice display altered emotional responses," Proceedings of the National Academy of Sciences of the United States of America, vol. 98, no. 4, pp. 19581963, 2001.

[43] A. Asok, L. W. Ayers, B. Awoyemi, J. Schulkin, and J. B. Rosen, "Immediate early gene and neuropeptide expression following exposure to the predator odor 2,5-dihydro-2,4,5trimethylthiazoline (TMT)," Behavioural Brain Research, vol. 248, pp. 85-93, 2013.

[44] K. Szklarczyk, M. Korostynski, P. E. Cieslak, A. WawrzczakBargiela, and R. Przewlocki, "Opioid-dependent regulation of high and low fear responses in two inbred mouse strains," Behavioural Brain Research, vol. 292, pp. 95-101, 2015.

[45] W. Haubensak, P. S. Kunwar, H. Cai et al., "Genetic dissection of an amygdala microcircuit that gates conditioned fear," Nature, vol. 468, no. 7321, pp. 270-276, 2010.

[46] J. Hernández, I. Prieto, A. B. Segarra et al., "Interaction of neuropeptidase activities in cortico-limbic regions after acute restraint stress," Behavioural Brain Research, vol. 287, pp. 42-48, 2015.

[47] I. Liberzon, S. F. Taylor, K. L. Phan et al., "Altered central micro-opioid receptor binding after psychological trauma," Biological Psychiatry, vol. 61, no. 9, pp. 1030-1038, 2007.

[48] D. E. Comings, G. Dietz, R. Gade-Andavolu et al., "Association of the neutral endopeptidase (MME) gene with anxiety," Psychiatric Genetics, vol. 10, no. 2, pp. 91-94, 2000.

[49] S. E. Kennedy, R. A. Koeppe, E. A. Young, and J. K. Zubieta, "Dysregulation of endogenous opioid emotion regulation circuitry in major depression in women," Archives of General Psychiatry, vol. 63, no. 11, pp. 1199-1208, 2006.

[50] A. Bilkei-Gorzo, I. Racz, K. Michel, A. Zimmer, D. Klingmüller, and A. Zimmer, "Behavioral phenotype of pre-proenkephalindeficient mice on diverse congenic backgrounds," Psychopharmacology, vol. 176, no. 3-4, pp. 343-352, 2004.

[51] M. König, A. M. Zimmer, H. Steiner et al., "Pain responses, anxiety and aggression in mice deficient in pre-proenkephalin," Nature, vol. 383, no. 6600, pp. 535-538, 1996.
[52] J. C. Kung, T. C. Chen, B. C. Shyu, S. Hsiao, and A. C. Huang, "Anxiety- and depressive-like responses and c-fos activity in preproenkephalin knockout mice: oversensitivity hypothesis of enkephalin deficit-induced posttraumatic stress disorder," Journal of Biomedical Science, vol. 17, p. $29,2010$.

[53] D. Filliol, S. Ghozland, J. Chluba et al., "Mice deficient for delta- and mu-opioid receptors exhibit opposing alterations of emotional responses," Nature Genetics, vol. 25, no. 2, pp. 195-200, 2000.

[54] S. A. Perrine, B. A. Hoshaw, and E. M. Unterwald, "Delta opioid receptor ligands modulate anxiety-like behaviors in the rat," British Journal of Pharmacology, vol. 147, no. 8, pp. 864-872, 2006.

[55] D. C. Broom, E. M. Jutkiewicz, K. C. Rice, J. R. Traynor, and J. H. Woods, "Behavioral effects of delta-opioid receptor agonists: potential antidepressants?" Japanese Journal of Pharmacology, vol. 90, no. 1, pp. 1-6, 2002.

[56] A. Saitoh, Y. Kimura, T. Suzuki, K. Kawai, H. Nagase, and J. Kamei, "Potential anxiolytic and antidepressant-like activities of SNC80, a selective delta-opioid agonist, in behavioral models in rodents," Journal of Pharmacological Sciences, vol. 95, no. 3, pp. 374-380, 2004.

[57] R. Vergura, G. Balboni, B. Spagnolo et al., "Anxiolytic- and antidepressant-like activities of $\mathrm{H}$-Dmt-Tic- $\mathrm{NH}-\mathrm{CH}(\mathrm{CH} 2-$ $\mathrm{COOH}$ )-Bid (UFP-512), a novel selective delta opioid receptor agonist," Peptides, vol. 29, no. 1, pp. 93-103, 2008.

[58] A. Rezayof, S. S. Hosseini, and M. R. Zarrindast, "Effects of morphine on rat behaviour in the elevated plus maze: the role of central amygdala dopamine receptors," Behavioural Brain Research, vol. 202, no. 2, pp. 171-178, 2009.

[59] C. Nozaki, H. Nagase, T. Nemoto, A. Matifas, B. L. Kieffer, and C. Gaveriaux-Ruff, "In vivo properties of KNT-127, a novel delta opioid receptor agonist: receptor internalization, antihyperalgesia and antidepressant effects in mice," British Journal of Pharmacology, vol. 171, no. 23, pp. 5376-5386, 2014.

[60] A. Saitoh, A. Sugiyama, M. Yamada et al., "The novel delta opioid receptor agonist KNT-127 produces distinct anxiolytic-like effects in rats without producing the adverse effects associated with benzodiazepines," Neuropharmacology, vol. 67, pp. 485493, 2013.

[61] A. Sugiyama, H. Nagase, J. Oka, M. Yamada, and A. Saitoh, " $\mathrm{DOR}_{2}$-selective but not $\mathrm{DOR}_{1}$-selective antagonist abolishes anxiolytic-like effects of the delta opioid receptor agonist KNT-127," Neuropharmacology, vol. 79, pp. 314-320, 2014.

[62] P. Chu Sin Chung, H. L. Keyworth, E. Martin-Garcia et al., "A novel anxiogenic role for the delta opioid receptor expressed in GABAergic forebrain neurons," Biological Psychiatry, vol. 77, no. 4, pp. 404-415, 2015.

[63] L. Granholm, E. Roman, and I. Nylander, "Single housing during early adolescence causes time-, area- and peptidespecific alterations in endogenous opioids of rat brain," British Journal of Pharmacology, vol. 172, no. 2, pp. 606-614, 2015.

[64] W. Zhu and Z. Z. Pan, "Synaptic properties and postsynaptic opioid effects in rat central amygdala neurons," Neuroscience, vol. 127, no. 4, pp. 871-879, 2004.

[65] W. Zhu and Z. Z. Pan, "Mu-opioid-mediated inhibition of glutamate synaptic transmission in rat central amygdala neurons," Neuroscience, vol. 133, no. 1, pp. 97-103, 2005. 
[66] S. Ciocchi, C. Herry, F. Grenier et al., "Encoding of conditioned fear in central amygdala inhibitory circuits," Nature, vol. 468, no. 7321, pp. 277-282, 2010.

[67] I. Melo, E. Drews, A. Zimmer, and A. Bilkei-Gorzo, "Enkephalin knockout male mice are resistant to chronic mild stress," Genes, Brain, and Behavior, vol. 13, no. 6, pp. 550-558, 2014.

[68] S. J. Russo, J. W. Murrough, M. H. Han, D. S. Charney, and E. J. Nestler, "Neurobiology of resilience," Nature Neuroscience, vol. 15, no. 11, pp. 1475-1484, 2012.

[69] B. M. Sweis, K. K. Veverka, E. S. Dhillon, J. H. Urban, and L. R. Lucas, "Individual differences in the effects of chronic stress on memory: behavioral and neurochemical correlates of resiliency," Neuroscience, vol. 246, pp. 142-159, 2013.

[70] J. F. Poulin, S. Laforest, and G. Drolet, "Enkephalin downregulation in the nucleus accumbens underlies chronic stress-induced anhedonia," Stress, vol. 17, no. 1, pp. 88-96, 2014.

[71] L. A. Briand, M. Hilario, H. C. Dow, E. S. Brodkin, J. A. Blendy, and O. Berton, "Mouse model of OPRM1 (A118G) polymorphism increases sociability and dominance and confers resilience to social defeat," The Journal of Neuroscience, vol. 35, no. 8, pp. 3582-3590, 2015.

[72] B. A. Reyes, G. Zitnik, C. Foster, E. J. Van Bockstaele, and R. J. Valentino, "Social stress engages neurochemicallydistinct afferents to the rat locus coeruleus depending on coping strategy," eNeuro, vol. 2, no. 6, 2015.

[73] V. Vialou, A. J. Robison, Q. C. Laplant et al., "DeltaFosB in brain reward circuits mediates resilience to stress and antidepressant responses," Nature Neuroscience, vol. 13, no. 6, pp. 745-752, 2010.

[74] H. Akil, S. J. Watson, E. Young, M. E. Lewis, H. Khachaturian, and J. M. Walker, "Endogenous opioids: biology and function," Annual Review of Neuroscience, vol. 7, pp. 223-255, 1984.

[75] S. A. Golden, H. E. Covington 3rd, O. Berton, and S. J. Russo, "A standardized protocol for repeated social defeat stress in mice," Nature Protocols, vol. 6, no. 8, pp. 11831191, 2011.

[76] S. Bhatnagar and C. Vining, "Facilitation of hypothalamicpituitary-adrenal responses to novel stress following repeated social stress using the resident/intruder paradigm," Hormones and Behavior, vol. 43, no. 1, pp. 158-165, 2003.

[77] R. Rygula, N. Abumaria, G. Flügge, E. Fuchs, E. Rüther, and U. Havemann-Reinecke, "Anhedonia and motivational deficits in rats: impact of chronic social stress," Behavioural Brain Research, vol. 162, no. 1, pp. 127-134, 2005.

[78] M. Peciña, T. Love, C. S. Stohler, D. Goldman, and J. K. Zubieta, "Effects of the mu opioid receptor polymorphism (OPRM1 A118G) on pain regulation, placebo effects and associated personality trait measures," Neuropsychopharmacology, vol. 40, no. 4, pp. 957-965, 2015.

[79] A. Troisi, G. Frazzetto, V. Carola et al., "Social hedonic capacity is associated with the A118G polymorphism of the muopioid receptor gene (OPRM1) in adult healthy volunteers and psychiatric patients," Social Neuroscience, vol. 6, no. 1, pp. 88-97, 2011.

[80] A. Troisi, G. Frazzetto, V. Carola et al., "Variation in the muopioid receptor gene (OPRM1) moderates the influence of early maternal care on fearful attachment," Social Cognitive and Affective Neuroscience, vol. 7, no. 5, pp. 542-547, 2012.
[81] M. S. Henry, K. Bisht, N. Vernoux, L. Gendron, M. Tremblay, and G. Drolet, "Enkephalin and Delta opioid receptor promote the resilience to chronic stress," in Program No. 814.23. 2016 Neuroscience Meeting Planner of Society for Neuroscience, 2016 (Online), San Diego, CA, 2016.

[82] J. F. Randall-Thompson, K. A. Pescatore, and E. M. Unterwald, "A role for delta opioid receptors in the central nucleus of the amygdala in anxiety-like behaviors," Psychopharmacology, vol. 212, no. 4, pp. 585-595, 2010. 

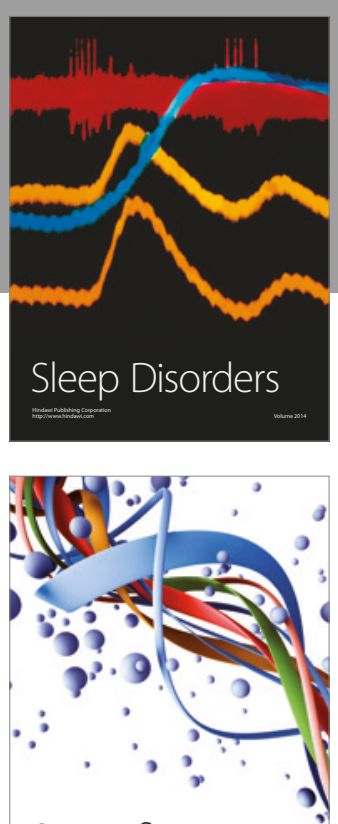

Scientifica
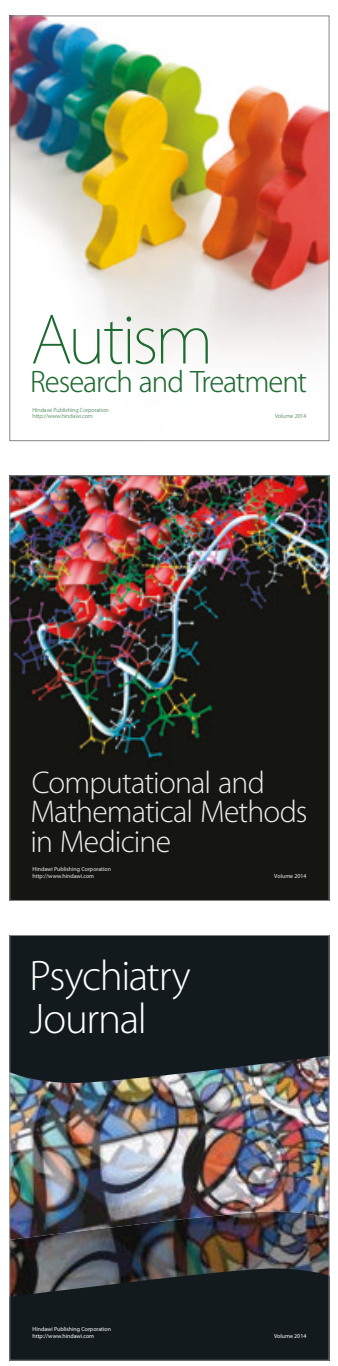
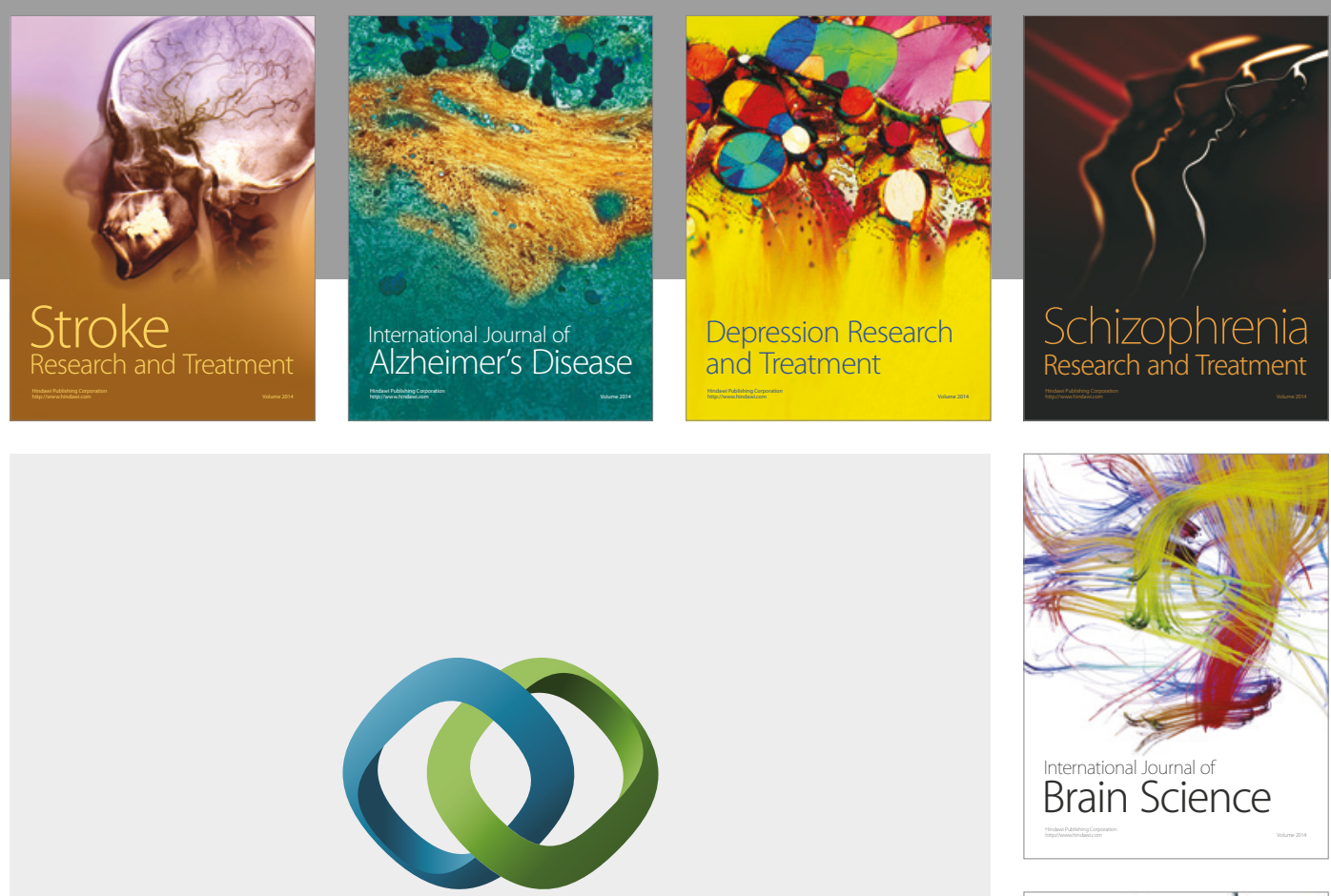

\section{Hindawi}

Submit your manuscripts at

https://www.hindawi.com
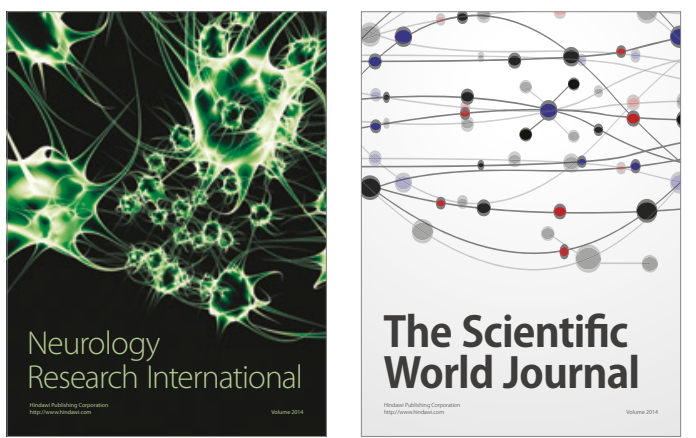

The Scientific World Journal

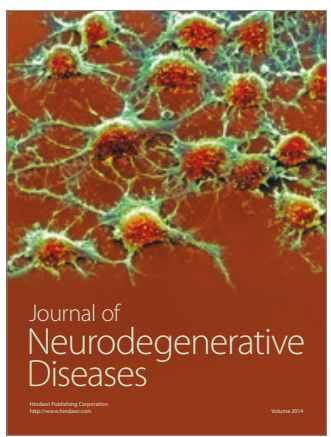

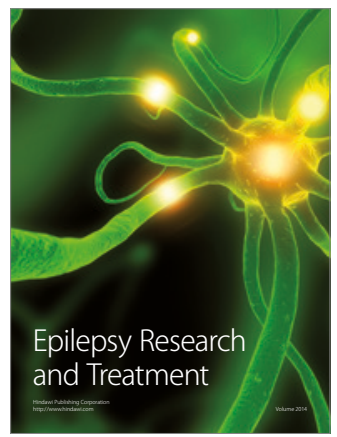

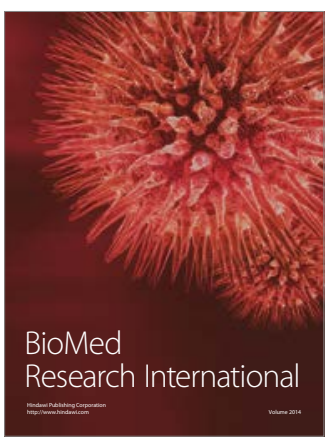

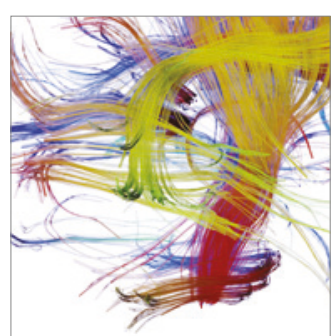

Brain Science

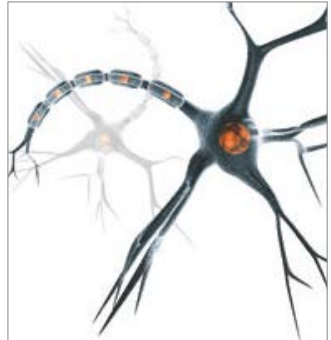

Neural Plasticity
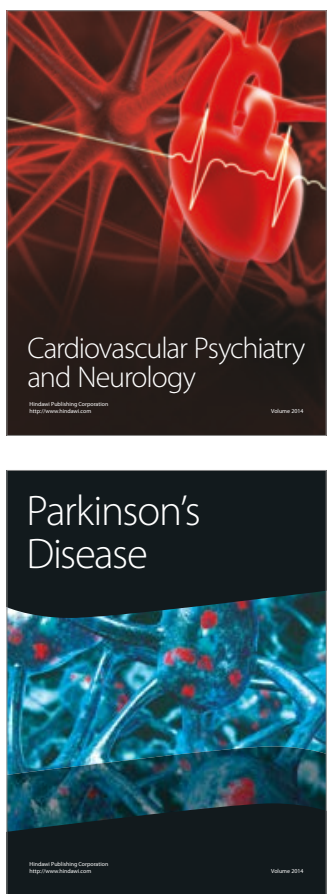